\title{
An Optimized Runge-Kutta Method for the Numerical Solution of the Oscillatory Problems
}

\author{
${ }^{1}$ Firas A. Fawzi, ${ }^{1}$ A.M. Gaftan, ${ }^{2}$ P.E. Oguntunde and ${ }^{3}$ N. Senu \\ ${ }^{1}$ Department of Mathematics, Faculty of Computer Science and Mathematics, \\ Tikrit University, Tikrit, Iraq \\ ${ }^{2}$ Department of Mathematics, Covenant University, Ota, Nigeria \\ ${ }^{3}$ Department of Mathematics and Institute for Mathematical Research, \\ Universiti Putra Malaysia (UPM) 43400 Serdang, Selangor, Malaysia, firasadi101@gmail.com
}

\begin{abstract}
In this study, an optimized explicit Runge-Kutta (RK) method which is based on a method of Dormand with six-stage and fifth algebraic order with FSAL property denoted as the ORK 5 method is constructed. The proposed method solves first-order Ordinary Differential Equations (ODEs) by first converting the second order ODEs to an equivalent first order. The new method has zero phase-lag, zero amplification error and zero first derivative of the phase-lag. Absolute stability of the new method is as well shown. The numerical experiments are carried out to show the efficiency of the derived method in comparison with other existing RK methods.
\end{abstract}

Key words: Amplification error, numerical analysis, ordinary differential equation, oscillatory problems, phase lag, Runge-Kutta method

\section{INTRODUCTION}

In this study, we are dealing with the Initial Value Problems (IVPs) of the form:

$$
\begin{gathered}
y^{\prime}(x)=f(x, y), y\left(x_{0}\right)=y_{0}, \\
y^{\prime}\left(x_{0}\right)=y_{0}^{\prime} x \in[a, b]
\end{gathered}
$$

Where:

$$
\begin{aligned}
y(x) & =\left[y_{1}(x), y_{2}(x), \ldots, y_{s}(x)\right]^{T} \\
f(x, y) & =\left[f_{1}(x, y), f_{2}(x, y), \ldots, f_{s}(x, y)\right]^{T}
\end{aligned}
$$

$\mathrm{y}_{0}$ is a given vector of initial conditions and their solution is oscillatory. This type of problem occurs in various applied fields such as quantum mechanics, electronics, physical chemistry, molecular dynamics astronomy, chemical physics and control engineering.

In effect, Eq. 1 can be solved using Runge-Kutta methods or multi-step methods. The solution of Eq. 1 often shows a pronounced oscillatory behavior. In general, most problems with oscillatory or periodical behavior are a second order or higher order. Hence, it is important to reduce the higher order problems to first-order problems in order to solve the ODEs in Eq. 1. Several researchers have improved numerical methods for solving oscillatory problems based on the phase-fitted and amplification fitted properties. Simos and Aguiar (2001) constructed a modified phase-fitted RK method with phase-lag of order infinity for the numerical solution of periodic IVPs based on the fifth algebraic-order RK method of Dormand and Prince.

Chen et al. (2012) improved traditional RK methods by introducing frequency-depending weights in the update. With the phase-fitting and amplification-fitting conditions and algebraic order conditions, new practical RK integrators are obtained and two of the new methods have updates that are also phase-fitted and amplification-fitted. With the evolution of RK methods, Papadopoulos et al. (2010) developed a new Runge-Kutta Nystrom (RKN) method for the numerical solution of the Schrodinger equation with phase-lag and amplification error of order infinity based on the fourth-order RKN method by Dormand, El-Mikkawy and Prince. Meanwhile, Moo et al. (2013) derived two new RKN methods for solving second-order differential equations with oscillatory solutions based on two existing RKN methods, a fourth-order three-stage Garcias RKN method and fifth-order four-stage Hairers RKN method.

The derived methods both have two variable coefficients with zero amplification error (zero dissipative) and phase-lag of order infinity. In the last few years, Senu et al. (2014) constructed zero dissipative explicit RK

Corresponding Author: Firas A. Fawzi, Department of Mathematics, Faculty of Computer Science and Mathematics, Tikrit University, Tikrit, Iraq, firasadi101@gmail.com 
method for solving second-order ODEs with periodical solutions which have algebraic order three with the dissipation of order infinity. Fawzi et al. (2016a, b) developed fourth-algebraic-order phase-fitted and amplification-fitted modified RK method and fourth-order seven-stage phase-fitted and amplification-fitted RK methods, respectively. Hussain et al. (2016) proposed a new optimized RK method for solving oscillatory problems. Recently, Ahmad et al. (2017) constructed a phase-fitted and amplification-fitted two-derivative RK method of high algebraic order for the numerical solution of first-order Initial Value Problems (IVPs) which possesses oscillatory solutions.

In this research, the new technique will be constructed by combining the nullification of phase-lag amplification factor and phase-lags derivative, based on a method of Dormand with six-stage and fifth algebraic order (Butcher and Wanner, 1996).

\section{MATERIALS AND METHODS}

Phase lag analysis of Runge-Kutta method: We consider the m-stage explicit RK method of the form:

$$
\begin{gathered}
y_{n+1}=y_{n}+h \sum_{i=1}^{m} b_{i} k_{i} \\
k_{i}=f\left(x_{n}+c_{i} h, y_{n}+h \sum_{j=1}^{i-1} a_{i j} k_{j}\right) ; i=1, \ldots, m
\end{gathered}
$$

The method is said to be explicit when $a_{i j}=0$ for $i \leq j$ and implicit otherwise. The method in Eq. 2 and 3 can be reduced into Butcher tableau form (Fig. 1).

To derive the new method based on phase lag analysis, we consider the following test equation based on Moo et al. (2013):

$$
\mathrm{y}^{\prime}=\mathrm{ivy}
$$

where, $\mathrm{v}$ is real. Then, we compare the theoretical solution and the numerical solution for this equation. By requiring that the solutions are in phase with a maximal order in the step-size $h$, we derive the so-called dispersion relation. Applying the above method in Eq. 2 and 3 to the test Eq. 4, we obtain:

$$
\mathrm{y}_{\mathrm{n}}=\mathrm{a}_{*}^{\mathrm{n}} \mathrm{y}_{0}
$$

with:

$$
\mathrm{a}_{*}=\mathrm{A}_{\mathrm{m}}\left(\mathrm{H}^{2}\right)+\mathrm{iHB}_{\mathrm{m}} \mathrm{H}^{2}
$$

where, $H=$ vh. The amplification factor is, $a_{*}=a_{*}(H)$ and denotes the approximation to $\mathrm{y}\left(\mathrm{x}_{\mathrm{n}}\right)$. A comparison of

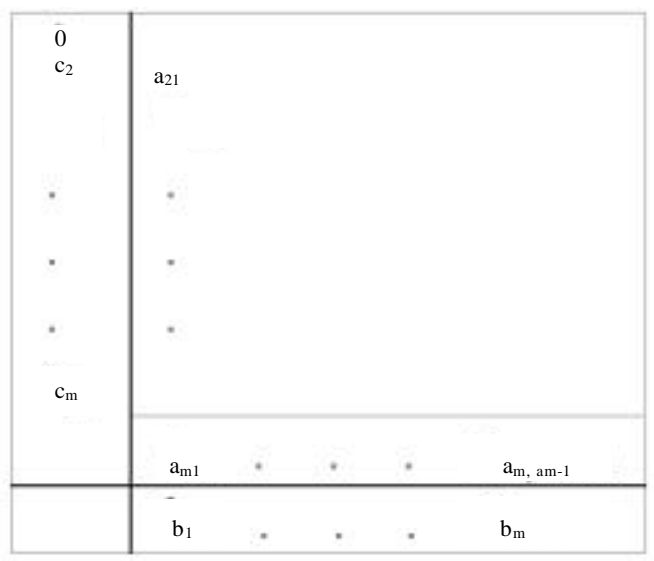

Fig. 1: m-stage explicit Runge-Kutta method

Eq. 5 with the solution of Eq. 4 leads to the following definition of the dispersion or phase error or phase-lag and the amplification error.

Definition 2.1: An explicit m-stage RK, presented in Fig. 1 the quantities:

$$
\begin{aligned}
& t(H)=H-\arg \left[a_{*}(H)\right] \\
& a(H)=1-\left|a_{*}(H)\right|
\end{aligned}
$$

are called the dispersion or phase error or phase-lag and the amplification error, respectively. If $\mathrm{t}(\mathrm{H})=\mathrm{O}\left(\mathrm{H}^{\mathrm{r}+1}\right)$ and $\mathrm{a}(\mathrm{H})=\mathrm{O}\left(\mathrm{H}^{\mathrm{s}+1}\right)$ then the method is said to be phase-lag order $\mathrm{r}$ and dissipative orders (Fawzi et al., 2016a, b). From Eq. 6, it follows that:

$$
\mathrm{a}(\mathrm{H})=1-\sqrt{\left[\mathrm{A}_{\mathrm{m}}^{2}\left(\mathrm{H}^{2}\right)+\mathrm{H}^{2} \mathrm{~B}_{\mathrm{m}}^{2}\left(\mathrm{H}^{2}\right)\right]}
$$

Meanwhile, for the Runge-Kutta method given in Fig. 1, the following formula is used for the direct calculation of the phase-lag order $r$ and the phase-lag constant q:

$$
\tan (\mathrm{H})-\mathrm{H}\left[\frac{\mathrm{B}_{\mathrm{m}}\left(\mathrm{H}^{2}\right)}{\mathrm{A}_{\mathrm{m}}\left(\mathrm{H}^{2}\right)}\right]=\mathrm{qH} \mathrm{H}^{\mathrm{r}+1}+\mathrm{O}\left(\mathrm{H}^{\mathrm{s}+3}\right)
$$

The analysis of phase-fitted (dispersion of order infinity) and amplification-fitted (dissipation of order infinity) are based on dispersion and dissipation quantities that have discussed above. The RK method is phase-fitted and amplification-fitted, if the following conditions hold:

$$
\mathrm{t}(\mathrm{H})=0 \text { and } \mathrm{a}(\mathrm{H})=0
$$


Table 1: Runge-Kutta method of order five

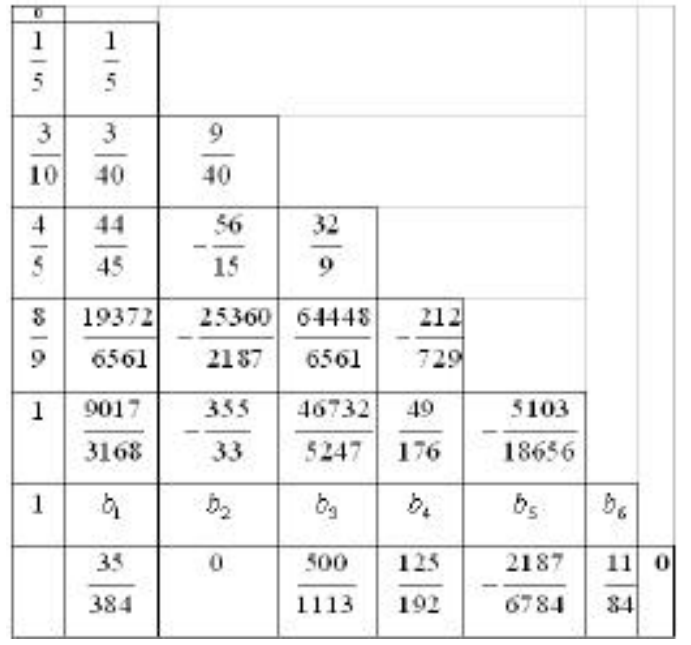

\section{RESULTS AND DISCUSSION}

Construction of the new Runge-Kutta methods: In this study, an optimized Runge-Kutta method will be derived, based on the fifth-order Runge-Kutta method with six-stage derived by Butcher and Wanner (1996) which is given in the tableau in Table 1. To achieve this, we set $b_{1}, b_{2}$ and $b_{3}$ as free coefficients while all other coefficients are the same as in Table 1, first, we compute the polynomials $\mathrm{A}_{\mathrm{m}}^{2}$ and $\mathrm{B}_{\mathrm{m}}^{2}$ in terms of Runge-Kutta coefficients in Table 1. Then from these polynomials, we obtain the quantities $t(H)$ and $a(H)$ and by the nullification of the phase-lag amplification error and phase-lag's derivative. Hence, we obtain a system of three equations as follows:

$$
\begin{gathered}
\mathrm{a}(\mathrm{H})=\left(1-\frac{1}{600} \mathrm{H}^{4}+\mathrm{QH}^{2}\right)+ \\
\mathrm{H}^{2}\left(\frac{1}{120} \mathrm{H}^{4}+\mathrm{PH}^{2}+\mathrm{b}_{3}+\mathrm{b}_{1}+\frac{65479}{142464}+\mathrm{b}_{2}\right)^{2}-1=0 \\
\mathrm{t}(\mathrm{H})=\tan (\mathrm{H})-\mathrm{H}\left(\frac{1}{120} \mathrm{H}^{4}+\mathrm{PH}^{2}+\mathrm{b}_{3}+\mathrm{b}_{1}+\frac{65479}{142464}+\mathrm{b}_{2}\right) \\
\left(1-\frac{1}{600} \mathrm{~h}^{6}+\frac{1}{24} \mathrm{~h}^{4}+\mathrm{Qh}^{2}\right)^{-1}=0 \\
\mathrm{t}^{\prime}(\mathrm{H})=1+[\tan (\mathrm{H})]- \\
\left(\frac{1}{120} \mathrm{H}^{4}+\mathrm{PH}^{2}+\mathrm{b}_{3}+\mathrm{b}_{1}+\frac{65479}{142464}+\mathrm{b}_{2}\right) \\
\left(1-\frac{1}{600} \mathrm{H}^{6}+\frac{1}{24} \mathrm{H}^{4}+\mathrm{QH}^{2}\right)^{-1}-\mathrm{H}\left(\frac{1}{30} \mathrm{H}^{3}+2 \mathrm{PH}\right)
\end{gathered}
$$

$$
\begin{aligned}
& \left(1-\frac{1}{600} \mathrm{H}^{6}+\frac{1}{24} \mathrm{H}^{4}+\mathrm{QH}^{2}\right)^{-1}+ \\
& \mathrm{H}\left(\frac{1}{120} \mathrm{H}^{4}+\mathrm{PH}^{2}+\mathrm{b}_{3}+\mathrm{b}_{1}+\frac{65479}{142464}+\mathrm{b}_{2}\right) \\
& \left(-\frac{1}{100} \mathrm{H}^{5}+\frac{1}{6} \mathrm{H}^{3}+2 \mathrm{QH}\right)\left(1-\frac{1}{600} \mathrm{H}^{6}+\frac{1}{24} \mathrm{H}^{4}+\mathrm{QH}^{2}\right)^{-2}
\end{aligned}
$$

where:

$$
\mathrm{P}=-\frac{163}{1113}-\frac{9}{200} \mathrm{~b}_{3}
$$

and:

$$
\mathrm{Q}=-\frac{3}{10} \mathrm{~b}_{3}-\frac{1}{5} \mathrm{~b}_{2}-\frac{271}{742}
$$

Solving simultaneously the system of Eq. 10-12, we obtain the coefficients $\mathrm{b}_{1}, \mathrm{~b}_{2}$ and $\mathrm{b}_{3}$ which are completely dependent on $\mathrm{H}$ where, $\mathrm{H}$ is the product of the step-size $h$ and the frequency v. The expressions for $b_{1}, b_{2}$ and $b_{3}$ are too complicated, hence, we replaced by their Taylor series expansion and obtained the following expressions:

$$
\begin{aligned}
& \mathrm{b}_{1}=\frac{35}{384}+\frac{643}{45360} \mathrm{H}^{4}+\frac{62677}{16329600} \mathrm{H}^{6}+\frac{5933}{4435200} \mathrm{H}^{8}+ \\
& \frac{50184187}{9340531200} \mathrm{H}^{10}+\frac{2560520257}{11769069312000} \mathrm{H}^{12}+ \\
& \mathrm{b}_{2}=-\frac{601}{15120} \mathrm{H}^{4}-\frac{1831}{217728} \mathrm{H}^{6}-\frac{26041}{798330} \mathrm{H}^{8}- \\
& \frac{328333}{249080832} \mathrm{H}^{10}-\frac{83804419}{156920924160} \mathrm{H}^{12}+\ldots, \\
& \mathrm{b}_{3}=\frac{500}{1113}+\frac{29}{1134} \mathrm{H}^{4}+\frac{451}{81648} \mathrm{H}^{6}+\frac{2171}{997920} \mathrm{H}^{8}+ \\
& \frac{410413}{467026560} \mathrm{H}^{10}+\frac{20951107}{58845346560} \mathrm{H}^{12}+\ldots .
\end{aligned}
$$

Stability of the new method: Here, the linear stability of the method developed is analyzed. Consider to the test Eq. 4 where $v>0$, the exact solution of this equation with initial value $\mathrm{y}\left(\mathrm{x}_{0}\right)=\mathrm{y}_{0}$ satisfies:

$$
\mathrm{y}\left(\mathrm{x}_{0}+\mathrm{h}\right)=\mathrm{R}(\mathrm{H}) \mathrm{y}_{0}
$$

When applying Eq. 2-4:

$$
\begin{gathered}
\mathrm{y}_{\mathrm{n}+1}=\mathrm{R}(\mathrm{H}) \mathrm{y}_{0} \\
\mathrm{R}(\mathrm{H})=1+\mathrm{Hb}^{\mathrm{T}}(\mathrm{I}-\mathrm{HA})^{-1} \mathrm{e}
\end{gathered}
$$

where, $\mathrm{e}=(1, \ldots, 1)^{\mathrm{T}}, \mathrm{A}=\left[\mathrm{a}_{\mathrm{ij}}\right]$ and $\mathrm{b}^{\mathrm{T}}=\left[\mathrm{b}_{1}, \mathrm{~b}_{2}, \mathrm{~b}_{3}, \ldots, \mathrm{b}_{\mathrm{m}}\right]$. $\mathrm{R}(\mathrm{H})$ is called the stability function of the method in Eq. 3 . 
Definition 3.1: A Runge-Kutta method is said to be absolutely stable if $\forall \mathrm{H} \in(-\mathrm{h}, 0),\left|\mathrm{R}\left(\mathrm{H}^{\wedge}\right)\right|<1$ (Fawzi et al., $2016 a, b)$. The stability polynomial of the ORK 5 method is given as follows:

$$
\begin{aligned}
& \mathrm{R}(\mathrm{H})=1+\mathrm{H}+\frac{1}{2} \mathrm{H}^{2}+\frac{1}{6} \mathrm{H}^{3}+\frac{1}{24} \mathrm{H}^{4}+\frac{1}{120} \mathrm{H}^{5}+ \\
& \frac{1}{720} \mathrm{H}^{6}+\frac{53}{25200} \mathrm{H}^{7}-\frac{1}{40320} \mathrm{H}^{8}+\frac{907}{1814400} \mathrm{H}^{9}+ \\
& \frac{1}{3628800} \mathrm{H}^{10}+\frac{39073}{199584000} \mathrm{H}^{11}-\frac{1}{479001600} \mathrm{H}^{12}+ \\
& \frac{2462483}{31135104000} \mathrm{H}^{13}+\frac{1}{87178291200} \mathrm{H}^{14}+ \\
& \frac{9164119691}{57164050944000} \mathrm{H}^{15}+\frac{11547559819}{266765571072000} \mathrm{H}^{16}+ \\
& \frac{11547559819}{1778437140480000} \mathrm{H}^{17}
\end{aligned}
$$

The comparison of the stability region of the ORK5 method up to $\mathrm{H}^{\mathrm{i}}$ where, $\mathrm{I}=8,10,12$ and its original method is plotted in Fig. 2. The stability interval of the original method is -306567892 and the stability interval of this method with the coefficients of $\mathrm{H}^{8}, \mathrm{H}^{10}, \mathrm{H}^{12}$ is -3.306570336 . Observing from the stability regions plotted in Fig. 2, our new method is absolutely stable, since,

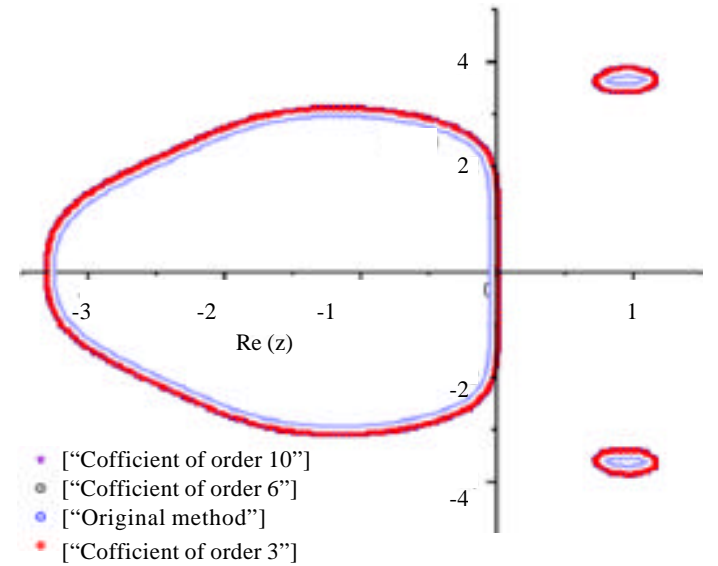

Fig. 2: Stability region of ORK 5 method for different order $\forall \mathrm{H} \in(-3.3,0)|\mathrm{R}(\mathrm{H})|<1$. We, however, obtained the result using maple package. Figure 2 shows the stability region of ORK 5 method for different order.

Error analysis: The Local Truncation Error (LTE) of the new method is based on the Taylor series expansion of the differences $y_{n+1}$ and $y\left(x_{n}+h\right)$ :

$$
\mathrm{LTE}=\mathrm{y}_{\mathrm{n}+1}+\mathrm{y}\left(\mathrm{x}_{\mathrm{n}}+\mathrm{h}\right)
$$

$$
\begin{aligned}
& \text { LTE }=h^{6}\left[\frac{1}{3600} w^{4} f_{y} f+\frac{2417}{381600} f_{x x x y y} f^{2}+\frac{21361}{3052800} f_{x x x y} f_{x}+\frac{1517}{381600} f_{x x y y y} f^{3}\right]+ \\
& h^{6}\left[\frac{35747}{6105600} f_{y x z} f_{x x}+\frac{239}{254400} f_{x y y y y} f^{4}+\frac{30045211}{6328115200} f_{x y y} f_{x z}+\frac{17563}{9158400} f_{z y} f_{x x z}\right]+ \\
& h^{6}\left[\frac{31}{8480} f_{x y y} f_{x}+\frac{1}{648000} f_{y y y y y} f^{5}+\frac{2251}{4070400} f_{y} f_{x x x z}-\frac{1}{3600} f_{y y y y} f_{x}-\frac{1}{3600} f_{y y y y y} f\right]+ \\
& \mathrm{h}^{6}\left[\frac{61187}{3052800} \mathrm{ff}_{\mathrm{zyy}} \mathrm{f}_{\mathrm{y}} \mathrm{f}_{\mathrm{x}}+\frac{213825583}{32036083200} \mathrm{f}_{\mathrm{xy}} \mathrm{ff}_{\mathrm{yy}} \mathrm{f}_{\mathrm{x}}+\frac{22403}{3434400} \mathrm{f}_{\mathrm{zy}} \mathrm{f}_{\mathrm{yy}} \mathrm{f}_{\mathrm{y}} \mathrm{f}^{2}\right]+ \\
& h^{6}\left[\frac{17641}{10074240} \mathrm{ff}_{\mathrm{yy}} \mathrm{f}_{\mathrm{yy}} \mathrm{f}_{\mathrm{z}}+\frac{27}{13568} \mathrm{f}^{2} \mathrm{f}_{\mathrm{yyy}} \mathrm{f}_{\mathrm{y}} \mathrm{f}_{\mathrm{x}}+\frac{6859}{1526400} \mathrm{f}_{\mathrm{xxxxy}} \mathrm{f}\right]+ \\
& h^{6}\left[\frac{14161}{1017600} f_{x x y y} f_{x}+\frac{94}{11925} f_{x x x y} f_{y} f+\frac{17}{1272} f_{x x y y} f_{y} f^{2}+\frac{343}{25440} f_{y x z} f_{x y} f\right]+ \\
& h^{6}\left[\frac{349}{47700} f_{y x x} f_{y y} f^{2}+\frac{2527}{190800} f_{y x x} f_{y} f_{x}+\frac{2527}{190800} f_{y x z} f_{y y} f+\frac{2317}{381600} f_{x y y y} f_{y} f^{3}\right]+ \\
& h^{6}\left[\frac{7649}{1017600} f_{x y y} f_{x x}+\frac{4979}{381600} f_{x y y} f_{x y} f^{2}+\frac{113}{21200} f_{x y y} f_{y y} f^{3}+\frac{11627}{763200} f_{x y y} f_{y y} f^{2}\right]+ \\
& h^{6}\left[\frac{2257}{381600} f_{x y y} f_{y} f+\frac{833}{429300} f_{x y} f_{y y y} f^{3}+\frac{431}{381600} f_{x y} f_{y} f_{x z}+\frac{333627097}{17085911040} f_{y y y} f_{x x}\right]+ \\
& h^{6}\left[\frac{217}{122112} \mathrm{ff}_{y y} \mathrm{f}_{\mathrm{xxx}}+\frac{18649}{27475200} \mathrm{f}^{3} \mathrm{f}_{y y y} \mathrm{f}_{\mathrm{z}}+\frac{1}{129600} \mathrm{f}_{y y y y} \mathrm{f}_{\mathrm{y}} \mathrm{f}^{4}+\frac{104123}{54950400} \mathrm{f}^{2} \mathrm{f}_{\mathrm{yyy}} \mathrm{f}_{\mathrm{xz}}\right]+ \\
& h^{6}\left[\frac{1}{64800} f_{y y y} f_{y y} f^{4}-\frac{1}{43200} f_{y y y} f_{y y} f^{3}+\frac{18649}{27475200} f^{2} f_{y y y} f_{z}+\frac{1}{64800} f_{y y y} f_{y} f^{3}\right]+
\end{aligned}
$$




$$
\begin{aligned}
& \mathrm{h}^{6}\left[\frac{11}{43200} \mathrm{f}_{\mathrm{yy}} \mathrm{f}_{\mathrm{yyy}} \mathrm{f}^{2}+\frac{1252708019}{512577331200} \mathrm{f}_{\mathrm{yy}} \mathrm{f}_{\mathrm{xz}} \mathrm{f}_{\mathrm{z}}+\frac{75379}{50371200} \mathrm{f}_{\mathrm{yy}} \mathrm{f}_{\mathrm{y}} \mathrm{f}_{\mathrm{zxz}}+\frac{73097}{6105600} \mathrm{f}_{\mathrm{xxxxz}}\right]+ \\
& \mathrm{h}^{6}\left[\frac{1}{3600} \mathrm{w}^{4} \mathrm{f}_{\mathrm{z}}+\frac{102427}{54950400} \mathrm{ff}_{\mathrm{yy}} \mathrm{f}_{\mathrm{y}} \mathrm{f}_{\mathrm{zxz}}+\frac{2587}{339200} \mathrm{f}^{2} \mathrm{f}_{\mathrm{xyyy}} \mathrm{f}_{\mathrm{x}}\right]+\mathrm{O}\left(\mathrm{h}^{7}\right)
\end{aligned}
$$

From Eq. 18, it is clear that the order of the new method is five because all the terms of $h$ lower than $\mathrm{h}^{6}$ are vanished.

Tested problems and numerical results: In this study, the performance of the proposed method ORK 5 is compared with existing RK methods by considering the following problems. All problems below are tested using $\mathrm{C}$ code for solving differential equations where the solutions are periodic:

ORK5: An optimized fifth-order RK method derived in this study.

MODRK5PLDPLAM: The phase-fitted six-stage fifth-order RK method derived by Ming et al. (2012).

MODPHARK5S: The modified phase-fitted fifth-order RK method given in Simos and Aguiar (2001 a, b).

PHRK54: The higher order method of the phase-fitted embedded RK5(4) proposed by Van de Vyver (2006).

RK-Fehlberg5th: An optimized fifth-order RK method derived by Kosti et al. (2010).

Problem 1: Homogeneous problem, Chawla and Rao (1985):

$$
\begin{array}{ll}
\mathrm{y}_{1}=\mathrm{y}_{2}, & \mathrm{y}_{1}(\mathrm{x})=1 \\
\mathrm{y}_{2}=-64 \mathrm{y}_{1}, & \mathrm{y}_{2}(\mathrm{x})=-2
\end{array}
$$

Theoretical solution:

$$
\begin{aligned}
& y_{1}(x)=-\frac{1}{4} \sin (8 x)+\cos (8 x) \\
& y_{2}(x)=-2 \cos (8 x)-8 \sin (8 x)
\end{aligned}
$$

Problem 2: In homogeneous problem, Van der Houwen and Sommeijer (1987):

$$
\begin{aligned}
& y_{1}=y_{2} \\
& y_{1}(x)=1 \\
& y_{2}=-v^{2} y_{1}+\left(v^{2}-1\right) \sin (x) \\
& y_{2}(x)=v+1
\end{aligned}
$$

Estimated frequency: $v=10$.

Theoretical solution:

$$
\begin{aligned}
& y_{1}(x)=\cos (v x)+\sin (v x)+\sin (x) \\
& y_{2}(x)=-v \sin (v x)+v \cos (v x)+\cos (x)
\end{aligned}
$$

Problem 3: Almost periodic orbit problem (Stiefel and Bettis, 1969):

$$
\begin{aligned}
& y_{1}=y_{3}, \quad y_{1}(x)=1 \\
& y_{3}=-y_{1}+0.001 \cos (x), \quad y_{3}(x)=0 \\
& y_{2}=y_{4}, \quad y_{2}(x)=0 \\
& y_{4}=-y_{2}+0.001 \sin (x), \quad y_{4}(x)=0.9995
\end{aligned}
$$

Theoretical solution:

$$
\begin{aligned}
& y_{1}(x)=\cos (x)+0.0005 x \sin (x) \\
& y_{2}(x)=\sin (x)-0.0005 x \cos (x) \\
& y_{3}(x)=-\sin (x)+0.0005 x \cos (x) \\
& y_{4}(x)=\cos (x)+0.0005 x \sin (x)
\end{aligned}
$$

Problem 4: Inhomogeneous system (Franco, 2006):

$$
\begin{aligned}
& \mathrm{y}_{1}=\mathrm{y}_{3}, \quad \mathrm{y}_{1}(\mathrm{x})=1 \\
& \mathrm{y}_{2}=-13 \mathrm{y}_{1}+12 \mathrm{y}_{2}+9 \cos (2 \mathrm{x})-12 \sin (2 \mathrm{x}) \\
& \mathrm{y}_{3}(\mathrm{x})=-4 \\
& \mathrm{y}_{3}=\mathrm{y}_{4}, \quad \mathrm{y}_{2}(\mathrm{x})=0 \\
& \mathrm{y}_{4}=12 \mathrm{y}_{1}-13 \mathrm{y}_{2}-12 \cos (2 \mathrm{x})+9 \sin (2 \mathrm{x}) \\
& \mathrm{y}_{4}(\mathrm{x})=8
\end{aligned}
$$

Estimated frequency: $v=5$

Theoretical solution:

$$
\begin{aligned}
& y_{1}(x)=\sin (x)-\sin (5 x)+\cos (2 x) \\
& y_{2}(x)=\sin (x)+\sin (5 x)+\sin (2 x) \\
& y_{3}(x)=\cos (x)-5 \cos (5 x)-2 \sin (2 x) \\
& y_{4}(x)=\cos (x)+5 \cos (5 x)+2 \cos (2 x)
\end{aligned}
$$

Problem 5: Inhomogeneous system, Salih et al. (2015): 


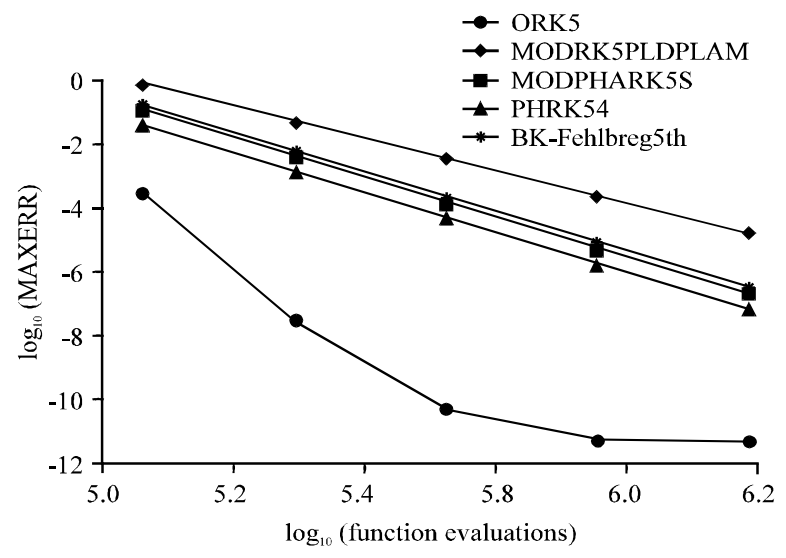

Fig. 3: Comparison for ORK5, MODRK5PLDPLAM, MODPHARK5S, PHRK54 and RK-Fehlberg 5th problem 1 with $b=10000$

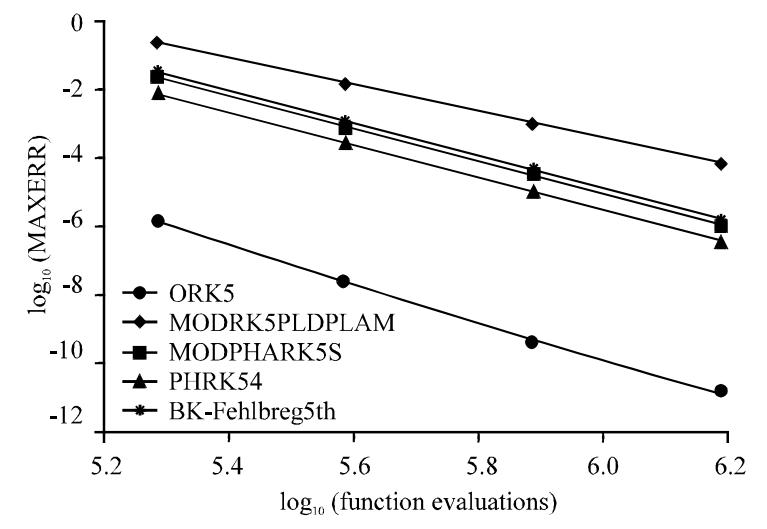

Fig. 4: Comparison for ORK5, MODRK5PLDPLAM, MODPHARK5S, PHRK 54 and RK-Fehlberg 5th problem 2 with $\mathrm{b}=10000$

$$
\begin{aligned}
& y_{1}^{\prime}=y_{3}, \quad y_{1}(x)=0 \\
& y_{2}^{\prime}=\frac{-101}{2} y_{1}+\frac{99}{2} y_{2}+\frac{93}{2} \cos (2 x)-\frac{99}{2} \sin (2 x) \\
& y_{3}(x)=-10 \\
& y_{3}^{\prime}=y_{4}, \quad y_{2}(x)=1 \\
& y_{4}^{\prime}=\frac{99}{2} y_{1}-\frac{101}{2} y_{2}+\frac{93}{2} \sin (2 x)-\frac{99}{2} \cos (2 x) \\
& y_{3}(x)=12
\end{aligned}
$$

Estimated frequency: $v=10$

Theoretical solution:

$$
\begin{aligned}
& \mathrm{y}_{1}(\mathrm{x})=-\cos (10 \mathrm{x})-\sin (10 \mathrm{x})+\cos (2 \mathrm{x}) \\
& \mathrm{y}_{2}(\mathrm{x})=\cos (10 \mathrm{x})+\sin (10 \mathrm{x})+\cos (2 \mathrm{x}) \\
& \mathrm{y}_{3}(\mathrm{x})=10 \sin (10 \mathrm{x})-10 \cos (10 \mathrm{x})-2 \sin (2 \mathrm{x}) \\
& \mathrm{y}_{4}(\mathrm{x})=-10 \sin (10 \mathrm{x})+10 \cos (10 \mathrm{x})+2 \cos (2 \mathrm{x})
\end{aligned}
$$

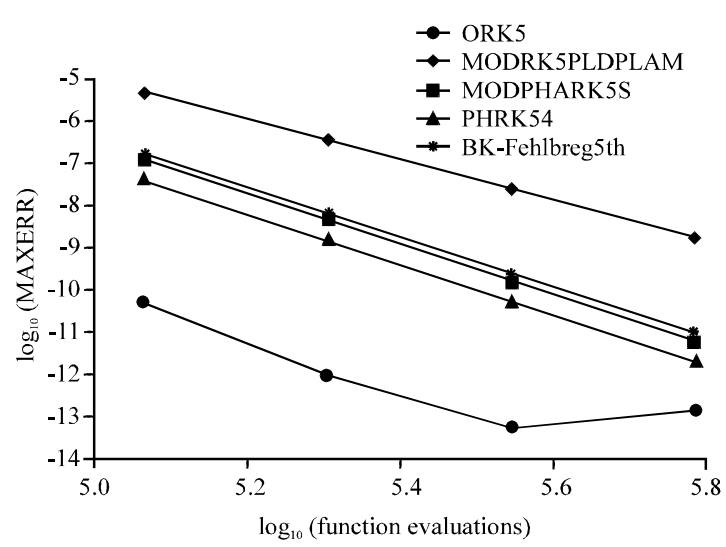

Fig. 5: Comparison for ORK5, MODRK5PLDPLAM, MODPHARK5S, PHRK54 and RK-Fehlberg 5th problem 3 with $b=10000$

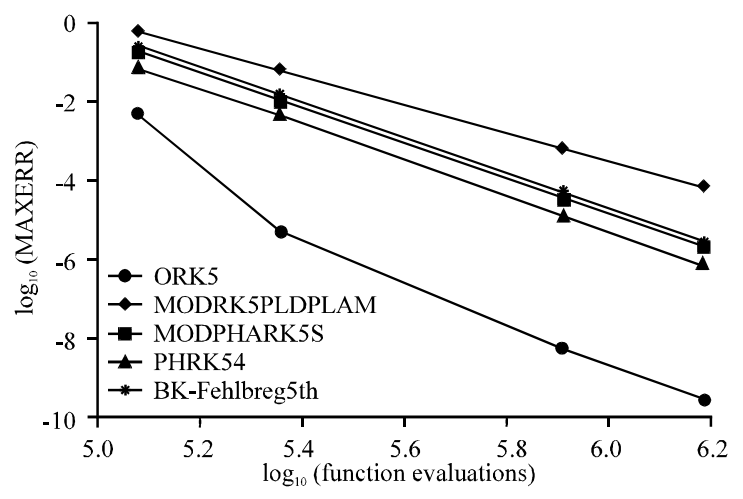

Fig. 6: Comparison for ORK5, MODRK5PLDPLAM, MODPHARK5S, PHRK54 and RK-Fehlberg 5th problem 4 with $b=10000$

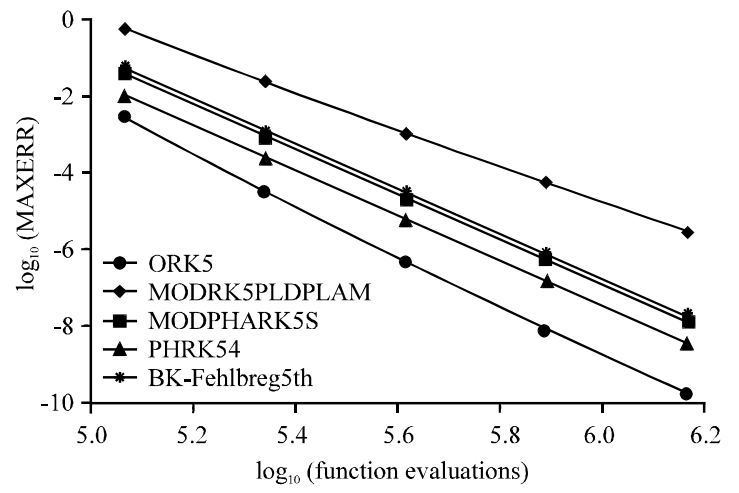

Fig. 7: Comparison for ORK5, MODRK5PLDPLAM, MODPHARK5S, PHRK54 and RK-Fehlberg 5th problem 5 with $\mathrm{b}=10000$

Figure 3-7 show the various comparisons between ORK5, MODRK5PLDPLAM, MODPHARK5S, PHRK54 and RK-Fehlberg5th problems 1-5, respectively with $\mathrm{b}=10000$. 


\section{CONCLUSION}

In this study, a six-stage fifth-order RK method denoted as ORK 5 for solving first-order ODEs by first converting the second order ODEs to an equivalent first order with phase-lag and amplification error of order infinity and the first derivative of phase-lag is of order infinity is developed. The comparison is made with other well-known existing explicit RK methods that have same algebraic order which are found in Van der Houwen and Sommeijer (1987), Ming et al. (2012), Simos and Aguiar (2001a, b) and Van de Vyver (2006). In the numerical comparisons, we used the criteria based on computing the maximum error in the solution [Max error $\left.=\max \left(\left|y\left(t_{n}-y_{n}\right)\right|\right)\right]$ which is equal to the maximum between absolute errors of the true solutions and the computed solutions. Figure 3-7 show the efficiency curves of $\log 10$ (max error) against the computational effort measured by $\log 10$ (function evaluations) required by each method and we observed that the new ORK5 method is more efficient for integration first-order differential equations possessing an oscillatory solution compared with other methods which are MODRK5PLDPLAM, MODPHARK5S, PHRK54 and RK-Fehlberg 5th.

\section{REFERENCES}

Ahmad, N.A., N. Senu and F. Ismail, 2017. Phase-fitted and amplification-fitted higher order two-derivative runge-kutta method for the numerical solution of orbital and related periodical IVPs. Math. Prob. Eng., 2017: 1-11.

Butcher, J.C. and G. Wanner, 1996. Runge-Kutta methods: Some historical notes. Appl. Numer. Math., 22: 113-151.

Chawla, M.M. and P.S. Rao, 1985. High-accuracy P-stable methods for $\mathrm{y}^{\prime \prime}=\mathrm{f}(\mathrm{t}, \mathrm{y})$. IMA. J. Numer. Anal., 5: 215-220.

Chen, Z., X. You, X. Shu and M. Zhang, 2012. A new family of phase-fitted and amplification-fitted Runge-Kutta type methods for oscillators. J. Appl. Math., 2012: 1-27.

Fawzi, F.A., N. Senu, F. Ismail and Z.A. Majid, 2016a. A new efficient phase-fitted and amplification-fitted runge-kutta method for oscillatory problems. Intl. J. Pure Appl. Math., 107: 69-86.

Fawzi, F.A., N. Senu, F. Ismail and Z.A. Majid, 2016b. New Phase-Fitted and amplification-fitted modified runge-kutta method for solving oscillatory problems. Global J. Pure Appl. Math., 12: 1229-1242.

Franco, J.M., 2006. A class of explicit two-step hybrid methods for second-order IVPs. J. Comput. Appl. Math., 187: 41-57.
Hussain, K., F. Ismail and N. Senu, 2016. A new optimized Runge-Kutta method for solving oscillatory problems. Intl. J. Pure Appl. Math., 106: 715-723.

Kosti, A.A., Z.A. Anastassi and T.E. Simos, 2010. An optimized explicit Runge-Kutta method with increased phase-lag order for the numerical solution of the Schrodinger equation and related problems. J. Math. Chem., 47: 315-330.

Ming, Q., Y. Yang and Y. Fang, 2012. An optimized Runge-Kutta method for the numerical solution of the radial Schrodinger equation. Math. Probl. Eng., 2012: 1-12.

Moo, K.W., N. Senu, F. Ismail and M. Suleiman, 2013. New phase-fitted and amplification-fitted fourth-order and fifth-order Runge-Kutta-Nystrom methods for oscillatory problems. Abstr. Appl. Anal., 2013: 1-9.

Papadopoulos, D.F., Z.A. Anastassi and T.E. Simos, 2010. A modified phase-fitted and amplification-fitted Runge-Kutta-Nystrom method for the numerical solution of the radial Schrodinger equation. J. Mol. Mod., 16: 1339-1346.

Salih, M., F. Ismail and N. Senu, 2015. Phase fitted classical runge-kutta method of order four for solving oscillatory problems. Far East J. Math. Sci., 96: 615-628.

Senu, N., I.A. Kasim, F. Ismail and N. Bachok, 2014. Zero-dissipative explicit Runge-Kutta method for periodic initial value problems. World Acad. Sci. Technol. Intl. J. Math. Comp. Phys. Electr. Comput. Eng., 8: 1226-1229.

Simos, T.E. and J.V. Aguiar, 2001b. A modified Runge-Kutta method with phase-lag of order infinity for the numerical solution of the Schrodinger equation and related problems. Comput. Chem., 25: 275-281.

Simos, T.E. and J.V. Aguiar, 2001a. A modified phase-fitted Runge-Kutta method for the numerical solution of the Schrodinger equation. J. Math. Chem., 30: 121-131.

Stiefel, E. and D.G. Bettis, 1969. Stabilization of Cowell's method. Numerische Math., 13: 154-175.

Van de Vyver, H., 2006. An embedded phase-fitted modified Runge-Kutta method for the numerical integration of the radial Schrodinger equation. Phys. Lett. A., 352: 278-285.

Van der Houwen, P.J. and B.P. Sommeijer, 1987. Explicit Runge-Kutta (-Nystrom) methods with reduced phase errors for computing oscillating solutions. SIAM. J. Numer. Anal., 24: 595-617. 